\title{
A pilgrim seeking diagnosis
}

\section{Aitor Uribarri, Iria A. González, Esther Pérez-David, Francisco Fernández-Avilés}

Cardiology Department, Hospital General Universitario “Gregorio Marañón”, Madrid, Spain

Email: auribarrig@gmail.com

Received 2 November 2012; revised 8 December 2012; accepted 14 December 2012

\begin{abstract}
We present the case of an unusual presentation of Dressler's syndrome and the important role of new imaging techniques in its diagnosis. A 42-year-old man was admitted with progressive dyspnoea and pleuritic chest pain during the last two months. Physical examination showed signs of hemodynamic instability (BP 75/40, HR 120 bpm). X-Ray showed cardiomegaly and electrocardiogram was suggestive of chronic anterior myocardial infarction. Transthoracic echocardiography showed pericardial effusion with signs of hemodynamic compromise. Emergent cardiac surgery was performed with suspicion of cardiac rupture. Only fibrinous material and serous fluid was found. 72 hours later he required reoperation because of recurrence of cardiac tamponade. Pleuropericardial window was made. Anti-inflammatory treatment was initiated. At discharge a magnetic resonance imaging study was performed. Diffuse pericardial inflammation consistent with a diagnosis of Dressler's syndrome (DS) was demonstrated in T2weighted black-blood and late enhancement sequences. One month later neither pleural nor pericardial effusion had recurred. Postinfarction pericardial effusions are common and magnetic resonance imaging has proven useful in determining its aetiology.
\end{abstract}

Keywords: Dressler's Syndrome; Postcardiac Injury Syndrome; Pericardial Effusion; Cardiac Tamponade; Magnetic Resonance Imaging

\section{BRIEF INTRODUCTION}

1956 William Dressler characterized a syndrome consisting of low-grade fever, pleuritic chest pain, pericardial friction rub and/or a pericardial effusion that rarely leads to cardiac tamponade. The symptoms tend to occur several weeks post myocardial infarction. Dressler's syndrome (DS) is also known as post-myocardial infarction syndrome (PMIS) and since the introduction of reperfusion its incidence has drastically decreased. Magnetic resonance imaging (MRI) may be useful when trying to establish the etiology of post-myocardial infarc- tion pericardial effusion.

\section{CASE DESCRIPTION}

We present the case of a 42-year-old man with progressive dyspnoea, pleuritic chest pain and low-grade fever during the last two months. He was admitted with evident signs of hemodynamic instability (BP 75/40, HR 120 bpm). Levels of troponin and creatine kinase were normal. Leucocytes were 24.100/mL (PMN 87\%), PCR 28 mg/dL, creatinin $1.9 \mathrm{mg} / \mathrm{dL}$ and INR blood test 1.45. X-Ray showed cardiomegaly and unilateral pleural effusion (Figure 1(a)) and electrocardiogram was suggestive of chronic anterior myocardial infarction. Echocardiography showed
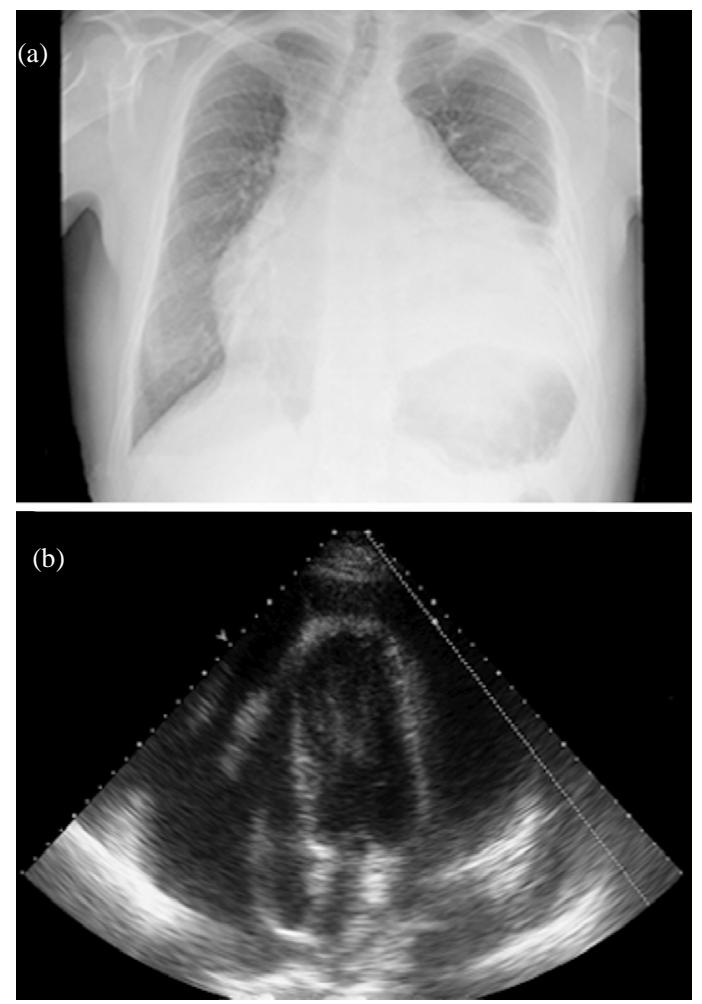

Figure 1. (a) Posteroanterior chest X-ray showed unilateral pleural effusion and cardiomegaly, suggesting pericardial effusion; (b) A large circunferential pericardial effusion $(>3 \mathrm{~cm})$ was seen in $2 \mathrm{D}$ echocardiography (4 chamber view). 
pericardial effusion (Figure 1(b)) with signs of hemodynamic compromise besides of severely impaired left ventricular function because of myocardial thinning and dyskinetic motion of anterior and apical segments. Emergent cardiac surgery was performed with suspicion of cardiac rupture. Only fibrinous material and serous fluid was found (Figure 2). Pleural fluid analysis was consistent with an exudative fluid with protein and LDH levels of $3.6 \mathrm{~g} / \mathrm{dL}$ and $201 \mathrm{UI} / \mathrm{L}$ (pleural fluid [LDH]/plasma $[\mathrm{LDH}]=0.75)$, respectively. The white blood cell count was $1500 / \mathrm{mcrl}$ with $52 \%$ of neutrophils, $\mathrm{pH}$ was 7.45 and the glucose was about the same as blood glucose levels. The cytology and the bacterial culture were negative. Pericardial biopsy specimens showed inflammatory infiltrate with histiocytes and neutrophils. 72 hours later he required reoperation because of recurrence of cardiac tamponade. Pleuropericardial window was made. Antiinflammatory treatment with acetylsalicylic acid and colchicine were initiated. Angiography showed subocclusion of anterior descending coronary artery so a coated metal stent was deployed with good result. CT scan was accomplished in order to rule out cancer or pulmonary embolism. At discharge a magnetic resonance imaging study was performed (Figures 3(a) and (b)). Diffuse pericardial inflammation consistent with a diagnosis of Dressler's syndrome (DS) was demonstrated in T2weighted black-blood (Figure 3(a)) and late enhancement sequences (Figure 3(b)). One month later the patient was free of dyspnoea and neither pleural nor pericardial effusion had recurred.

\section{DISCUSSION}

Dressler's syndrome (DS), or post-myocardial infarction syndrome (PMIS), is a secondary form of pericarditis that occurs in the setting of injury to the heart. The postcardiac injury syndrome (PCIS) is characterized by inflammation

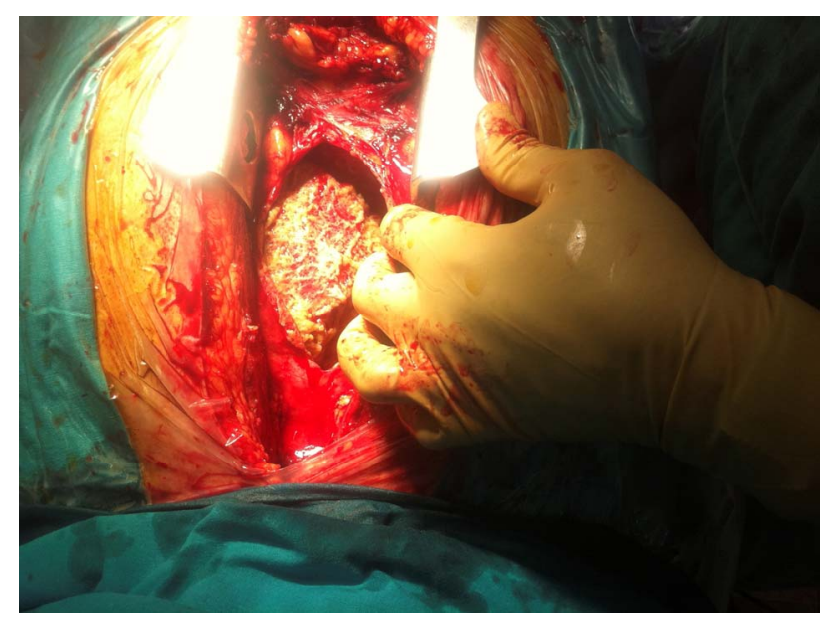

Figure 2. Image of cardiac surgery shows fibrinous material around the heart.
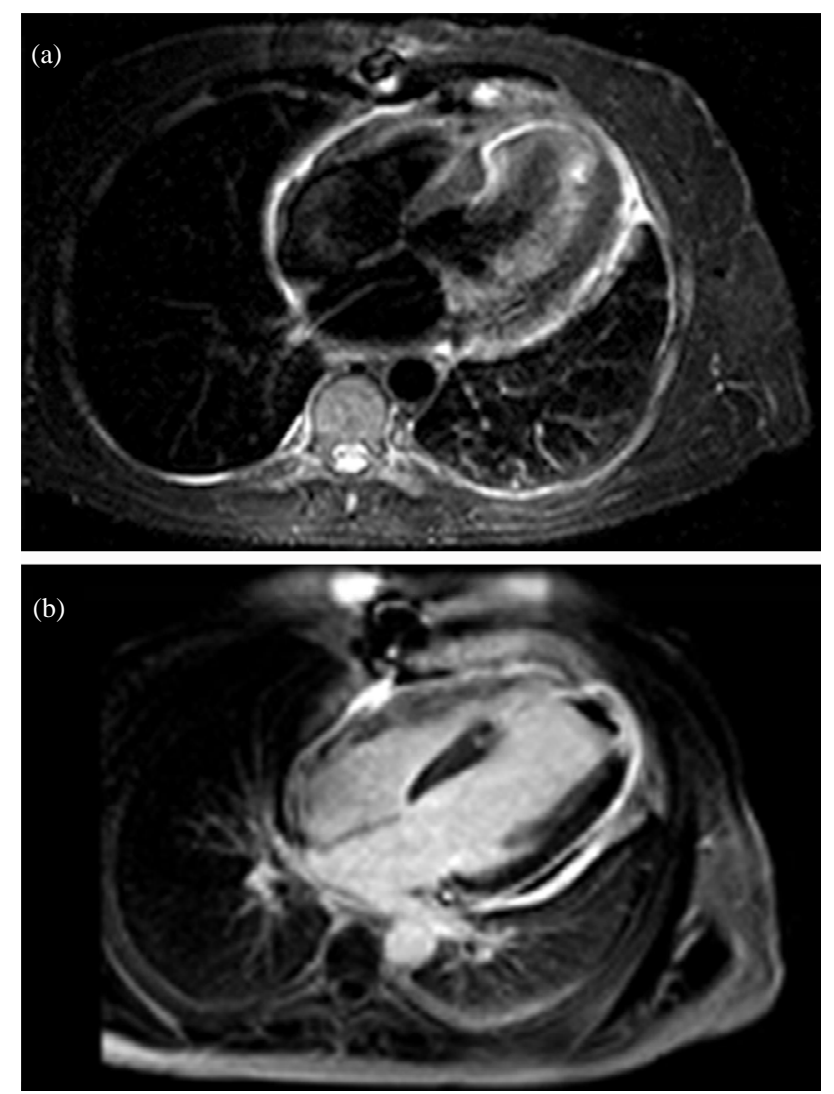

Figure 3. Image of cardiac surgery shows fibrinous material around the heart. (a) MRI short $\tau$-inversion recovery T2weighted images showed hyperintense signal from the pericardium, suggesting active inflammation, which was confirmed in late enhancement images; (b) Transmural enhancement was observed in LV distal septum, apex, and distal lateral wall, which was consistent with the diagnosis of anterior myocardial infarction. A non-enhanced area in the LV cavity corresponds to an apical thrombus.

of the pericardium, pleura and pulmonary parenchyma following a variety of cardiac injuries, and includes not only DS, but also postpericardiotomy syndrome (PPS) and it has been described following cardiac surgery, blunt chest trauma, percutaneous left ventricle puncture, implantation of a pacemaker, coronary angioplasty and radiofrequency ablation [1].

Dressler first described it in the 1950s as an uncommon complication of myocardial infarction. The syndrome consisted of one or more of these features: pericarditis, pleuritis and pneumonitis [2]. The symptoms tend to occur two weeks after the myocardial infarction, but can be delayed for a few months. First of all Dressler suggested a direct effect of blood in the pericardial cavity [2], while other authors supported a viral etiology [3]. Nowadays, it is believed to result from an autoimmune inflammatory reaction. There is evidence of complement activation and presence of autoantibodies [4].

In the last 50 years the incidence of DS has decreased 
from $5 \%$ to $0.5 \%$ [1]. The main reasons for this improvement are, firstly the reperfusion therapy has diminished cardiac tissue injury with reduction of antigen release into the bloodstream and secondly, antiplatelet drugs, ACE inhibitors, betablockers and statins, have proved their antiinflammatory and inmunomodulating properties [5].

DS consists of vague symptoms such as persistent fever, chest pain and dyspnoea. For diagnosis the following tests may be useful: in the laboratory tests stands out raised white blood-cell count and CRP, decreased C3 levels, presence of autoantibodies (antiheart, antiactin and antimyosin) and pleural and pericardial fluid analysis consistent with exudative fluid; electrocardiogram may present unusual evolutionary course showing ST segment reelevation; chest X-ray is abnormal in $95 \%$ of cases and pericardial effusion may be detected by echocardiogramphy [1]; CT scan is useful to rule out pulmonary embolism [1] and magnetic resonance imaging (MRI) may be crucial in differential diagnosis [6].

DS is typically treated with NSAIDs such as aspirin, and only with corticosteroids or colchicine if it is resistant to treatment [1]. This is an entity with favourable outcome not associated with an increase in mortality [1].

This case illustrates another clinical context where MRI may be useful when trying to establish the etiology of post-myocardial infarction pericardial effusion.

\section{REFERENCES}

[1] Wessman, D.E. and Stafford, C.M. (2006) The postcar- diac injury syndrome: Case report and review of the literature. Southern Medical Journal, 99, 309-314. doi:10.1097/01.smj.0000203330.15503.0b

[2] Dressler, W. (1956) A post-myocardial infarction syndrome: Preliminary report of a complication resembling idiopathic, recurrent, benign pericarditis. JAMA, 160, 1379-1383. doi:10.1001/jama.1956.02960510005002

[3] Burch, G.E. and Colcolough, H.L. (1970) Postcardiot- omy and postinfarction syndromes: A theory. American Heart Journal, 80, 290-291.

[4] McCabe, J.C., Ebert, P.A., Engle, M.A., et al. (1973) Circulating heart-reactive antibodies in the postpericardiotomy syndrome. Journal of Surgical Research, 14, 158164. doi:10.1016/0022-4804(73)90025-5

[5] Bendjelid, K. and Pugin, J. (2004) Is dressler syndrome dead? Chest, 126, 1680-1682. doi:10.1378/chest.126.5.1680

[6] Steadman, C.D., Khoo, J., Kovac, J. and McCann, G.P. (2009) Dressler's syndrome demonstrated by late gadolinium enhancement cardiovascular magnetic resonance. Journal of Cardiovascular Magnetic Resonance, 23, 1123. doi:10.1186/1532-429X-11-23 